Draft Version July 31, 2018

Typeset using LATEX twocolumn style in AASTeX61

\title{
OPTICAL EMISSION AND PARTICLE ACCELERATION IN A QUASI-STATIONARY COMPONENT IN THE JET OF OJ 287
}

\author{
Mahito Sasada, ${ }^{1,2,3}$ Svetlana Jorstad, ${ }^{3,4}$ Alan P. Marscher, ${ }^{3}$ Vishal Bala, ${ }^{3}$ Manasvita Joshi, ${ }^{3}$ \\ Nicholas R. MacDonald, ${ }^{3,5}$ Michael P. Malmrose, ${ }^{3}$ Valeri M. Larionov, ${ }^{4}$ Daria A. Morozova, 4 \\ Ivan S. Troitsky, ${ }^{4}$ Iván Agudo, ${ }^{6}$ Carolina Casadio, ${ }^{6}, 5$ José L. Gómez, ${ }^{6}$ Sol N. Molina, ${ }^{6}$ And Ryosuke Itoh ${ }^{7}$ \\ ${ }^{1}$ Hiroshima Astrophysical Science Center, Hiroshima University, 1-3-1 Kagamiyama, Higashi-Hiroshima, Hiroshima 739-8526, Japan \\ ${ }^{2}$ Mizusawa VLBI Observatory, NAOJ, 2-12 Hoshigaoka, Mizusawa-ku, Oshu, Iwate 023-0861, Japan \\ ${ }^{3}$ Institute for Astrophysical Research, Boston University, 725 Commonwealth Avenue, Boston, MA 02215, USA \\ ${ }^{4}$ Astronomical Institute, St. Petersburg University, Universitetskij Pr. 28, Petrodvorets, 198504, St. Petersburg, Russia \\ ${ }^{5}$ Max-Planck-Institute für Radioastronomie, Auf dem Hügel 69, D-53121 Bonn, Germany \\ ${ }^{6}$ Instituto de Astrofísica de Andalucía, CSIC, Apartado 3004, E-18080, Granada, Spain \\ ${ }^{7}$ Department of Physics, School of Science, Tokyo Institute of Technology, 2-12-1 Ohokayama, Meguro, Tokyo 152-8551, Japan
}

(Received August 17, 2017; Accepted July 20, 2018)

Submitted to ApJ

\begin{abstract}
We analyze the linear polarization of the relativistic jet in BL Lacertae object OJ 287 as revealed by multi-epoch Very Long Baseline Array (VLBA) images at $43 \mathrm{GHz}$ and monitoring observations at optical bands. The electric-vector position angle (EVPA) of the optical polarization matches that at $43 \mathrm{GHz}$ at locations that are often in the compact millimeter-wave "core" or, at other epochs, coincident with a bright, quasi-stationary emission feature $\sim 0.2$ milliarcsec ( $\sim 0.9 \mathrm{pc}$ projected on the sky) downstream from the core. This implies that electrons with high enough energies to emit optical synchrotron and $\gamma$-ray inverse Compton radiation are accelerated both in the core and at the downstream feature, the latter of which lies $\geq 10 \mathrm{pc}$ from the central engine. The polarization vector in the stationary feature is nearly parallel to the jet axis, as expected for a conical standing shock capable of accelerating electrons to GeV energies.
\end{abstract}

Keywords: galaxies: active - galaxies: jets - BL Lacertae objects: individual (OJ 287) - techniques: polarimetric - methods: observational 


\section{INTRODUCTION}

Production of the optical synchrotron radiation observed in blazars requires electrons with energies $\gtrsim 3 \mathrm{GeV}$. Similar electron energies are needed for inverse Compton scattering to generate the $\mathrm{GeV} \gamma$ ray emission often detected in the same objects (e.g., Marscher 2016). There are multiple possible sites in blazar jets where particles might be accelerated to such energies on (sub)parsec scales: (1) near the base of the jet, $\sim 20$ gravitational radii from the black hole (Hada et al. 2011), (2) in a magnetically dominated acceleration/collimation zone (ACZ) (Marscher et al. 2008, 2010; Sironi et al. 2015), (3) in regions where the plasma is turbulent, (4) in parsec-scale standing shocks (León-Tavares et al. 2010; Marscher 2014), and (5) in moving shocks that propagate from the jet base, through the parsec-scale "core" and beyond (e.g., Marscher \& Gear 1985; Joshi \& Böttcher 2011). Each of these possibilities corresponds to a distinct magnetic field geometry: helical field at the jet base, anti-parallel field lines in magnetic reconnection zones, disordered field lines in turbulent regions, and field parallel to shock fronts. Observations of the linear polarization of synchrotron radiation are sensitive probes of the magnetic field geometry, and therefore can test the various possibilities for particle acceleration.

In this study, we probe the magnetic field geometry in the millimeter-wave and optical emission zones of the BL Lacertae object OJ 287 (redshift $z=0.306$, at which 1 milliarcsecond $($ mas $)=4.5 \mathrm{pc}$; Nilsson et al. 2010) by combining multi-epoch total and polarized intensity Very Long Baseline Array (VLBA) images at $43 \mathrm{GHz}$ with well-sampled optical monitoring of the linear polarization. The VLBA images, with an angular resolution of $\sim 0.15$ mas, resolve the innermost regions of the jet. Comparison with the optical polarization allows us to determine the most likely site of optical synchrotron emission in the jet, as well as the geometry of the magnetic field in that location, at different times.

The compact jet of OJ 287 has changed dramatically over time (Agudo et al. 2012). Prior to 2005, it was directed to the southwest (Jorstad et al. 2005), in a similar direction as the arcsecond-scale radio/X-ray jet (Marscher \& Jorstad 2011). Its electric-vector position angle (EVPA) then abruptly switched by $\gtrsim 90^{\circ}$ to the north and then northwest (Agudo et al. 2011). Subsequently, a bright, quasi-stationary feature, " $S$ ", developed about 0.2 mas northwest of the bright compact "core" situated at the upstream end of the jet in $43 \mathrm{GHz}$ VLBA images(Agudo et al. 2011; Hodgson et al. 2017).

As is common for low-frequency-peaked BL Lac objects like OJ 287, the linear polarization at both mil- limeter and optical wavelengths is highly variable. For example, D'Arcangelo et al. (2009) measured changes from $9 \%$ to $15 \%$ in the degree of polarization of the core in $7 \mathrm{~mm}$ VLBA images, and from $8 \%$ to $30 \%$ at optical $R$-band during the same time span. Similarly, Villforth et al. (2010) reported optical polarization variations over a range from $\sim 0$ to $35 \%$ over $4.5 \mathrm{yr}$. This behavior provides the potential to locate the optically emitting region on VLBA images by matching the optical EVPA with that of a millimeter-wave feature (Marscher 2016). Here we apply this principle to OJ 287 over a period of nine years.

\section{OBSERVATIONS AND DATA ANALYSIS}

\subsection{VLBA Imaging and Optical Polarimetry}

OJ 287 is one of 37 blazars monitored roughly monthly at a wavelength of $7 \mathrm{~mm}$ with the VLBA under the VLBA-BU-BLAZAR project ${ }^{1}$ (Jorstad \& Marscher 2017). Calibration of the data, as well as total and polarized intensity imaging procedures, are essentially identical to those described by Jorstad et al. (2017) and Jorstad et al. (2007). In order to calibrate the absolute value of the EVPA of the VLBA data, we use (1) Very Large Array observations at $7 \mathrm{~mm}$ of a number of VLBA-BU-BLAZAR blazars, including OJ 287, within 1-3 days of the VLBA observations; and (2) the "D-terms" method (Gómez et al. 2001). We analyze Stokes-parameter $I, Q$, and $U$ images at 95 epochs from 2007 June 14 to 2016 March 18. Time intervals of VLBA observations are roughly 1 month, and most of them are within 2 months. All images are convolved with an elliptical Gaussian restoring beam of FWHM $0.33 \times 0.16$ mas oriented along position angle $\mathrm{PA}=-10^{\circ}$, approximating the angular resolution with uniform weighting of the visibilities. To check for signs of Faraday rotation and optical depth effects, we compared the EVPA in the $43 \mathrm{GHz}$ images when the EVPA was uniform across the main emission regions with a measurement of the integrated $86 \mathrm{GHz}$ polarization at the $30 \mathrm{~m}$ antenna of the Institut de Radioastronomie Millimétrique (Agudo et al. 2018a,b) within 2 days of the VLBA observation. Six epochs from 2008.50 to 2014.57 met these criteria. The mean 43-86 GHz EVPA difference was $2^{\circ} \pm 3^{\circ}$, leading us to conclude that the $43 \mathrm{GHz}$ polarization was unaffected by either Faraday rotation or synchrotron self-absorption.

We use data from optical polarization observations of OJ 287 performed at: (1) the $1.83 \mathrm{~m}$ Perkins Telescope of Lowell Observatory (Flagstaff, AZ) in $R$-band with

\footnotetext{
${ }^{1}$ http://www.bu.edu/blazars/VLBAproject.html
} 
the PRISM camera ${ }^{2}$; (2) the $0.7 \mathrm{~m}$ AZT-8 telescope of the Crimean Astrophysical Observatory (Nauchnij, Crimea) in $R$-band; (3) the $0.4 \mathrm{~m}$ LX-200 telescope of St. Petersburg University (St. Petersburg, Russia) without filter (central wavelength $\lambda_{\text {eff }} \approx 670 \mathrm{~nm}$ ); (4) the $1.54 \mathrm{~m}$ Kuiper and $2.3 \mathrm{~m}$ Bok telescopes of Steward Observatory (Tucson, AZ) in spectral-polarimetric mode covering a wavelength range of $4000-7550 \AA^{3}$; (5) the $2.2 \mathrm{~m}$ Telescope of Calar Alto Observatory (Almería, Spain) in $R$-band with the CAFOS multi-purpose focal reducer (acquired as part of the MAPCAT project ${ }^{4}$ ), and (6) the $1.5 \mathrm{~m}$ Kanata telescope of Higashi-Hiroshima Observatory (Hiroshima, Japan) in $V$-band. Details of the polarimetric data reduction can be found in Jorstad et al. (2010); Larionov et al. (2008); Smith et al. (2009); and Ikejiri et al. (2011). The differences in EVPA among the different optical bands are negligible compared with the temporal variations during the time spans between the observations. Finally, most of time intervals are within 1 -d in the time series of measured polarization.

We have examined the data sets to identify 43 pairs of VLBA and optical polarization observations simultaneous within $\pm 1 \mathrm{~d}$. The optical polarization measurements were collected nearly every night during periods when OJ 287 was visible. At epochs when multiple optical measurements matched the simultaneity criterion, we average the $I, Q$, and $U$ values to obtain the degree $P_{\mathrm{opt}}=\sqrt{Q^{2}+U^{2}} / I$ and position angle $\chi_{\mathrm{opt}}=\frac{1}{2} \tan ^{-1}\left(\frac{U}{Q}\right)$.

We employ the Gnu $R$ programming language (v3.2.1)(R Core Team 2015) for analysis of the VLBA images at these 43 epochs. At each epoch we determine $I_{\mathrm{i}}, Q_{\mathrm{i}}$, and $U_{\mathrm{i}}$ as the mean values over circular area i, $\mathrm{i}=1$ to $N$; the circular areas have radii of 0.05 mas and centers spaced every 0.05 mas along the jet trajectory. The averaged $N$ of 43 epochs is approximately 25. The degree of polarization $P_{\mathrm{i}}$ and EVPA $\chi_{\mathrm{i}}$ of each area are calculated from the corresponding Stokes parameters. The uncertainties $\delta I, \delta Q$, and $\delta U$ are derived as the mean values of the noise in eight off-source regions in each $I, Q$, and $U$ image. The uncertainties of $P_{\mathrm{i}}$ and $\chi_{\mathrm{i}}$ are calculated via standard propagation of errors (e.g., Kawabata et al. 1999). The results are corrected for Rice bias as described by Wardle \& Kronberg (1974), with data for which $P_{\mathrm{i}} / \delta P_{\mathrm{i}}<0.5$ excluded from consideration.

\footnotetext{
2 http://www.bu.edu/prism

3 http://james.as.arizona.edu/ psmith/Fermi/

${ }^{4}$ http://www.iaa.es/ iagudo/_iagudo/MAPCAT.html
}

\subsection{Determination of Jet Trajectory}

Optically thin synchrotron radiation has only a weak wavelength dependence of polarization, even as the spectral slope changes. The optically emitting region can, therefore, potentially be identified on VLBA images by comparing optical with radio polarization along the jet, provided that optical depth and Faraday effects are weak, as in OJ 287. In order to do this, we determine the jet trajectory in each VLBA image and calculate Stokes parameters $I, Q, U$ to determine the polarization vector at each step along the trajectory.

We perform the following procedures to determine the jet trajectory. (1) A line is drawn that roughly follows the jet axis on the VLBA image. (2) Line segments extending to \pm 0.8 mas from the axis are placed perpendicular to the local jet direction. The dashed and solid lines in panel (a) of Figure 1 illustrate this procedure. (3) The maximum intensity position is located on the solid line shown in panel (a) ${ }^{\prime}$ of Figure 1, which displays the intensity profile along the solid line. (4) The maximum intensity positions are determined every 0.01 or 0.02 mas, depending on the pixel size of the image. (5) A polynomial function corresponding to the jet trajectory is fit to these maximum intensity positions, as illustrated in panel (b) of Figure 1. We define the upstream boundary of the "core" of the jet as the position of the maximum gradient in the intensity profile along this trajectory.

The variation timescale of this object at optical wavelengths, $\delta \mathrm{t}$, is $\sim 0.8 \mathrm{~d}$, as determined by the $\mathrm{Ke}$ pler spacecraft (Goyal et al. 2017). Setting this to the light-crossing time allows us to estimate the size of the high-energy emitting zone as $\delta \mathrm{tc} D /(1+z) \sim 0.0046 \mathrm{pc}$, where $c$ is the speed of light and $D \sim 9$ is the Doppler factor (Jorstad et al. 2017). The spatial distance corresponding to the angular resolution of the VLBA image of 0.1 mas is $\sim 1.4 \mathrm{pc}\left(=\theta_{\mathrm{VLBA}} / \sin \Theta\right.$, where the angle between the jet axis and our line of sight, $\Theta$, is assumed to be $3^{\circ}$ ). The spatial distance is much larger than the size of the emitting zone estimated from the timescale of optical variations. Several optical emitting zones could therefore be contained within the spatial resolution of the VLBA.

We calculate the average $I, P$, and $\chi$ values within each circular region of radius 0.05 mas which is a half of the spatial resolution along the jet trajectory. Panels (d)-(f) of Figure 1 show an example of the distributions of the calculated intensity and polarization parameters. In this example, $\chi$ at $7 \mathrm{~mm}$ agrees well with that at optical wavelengths (solid line). The image and double-peaked polarization profile in Figure 1 reveal the presence of a component, " $S$," northwest of the core, 

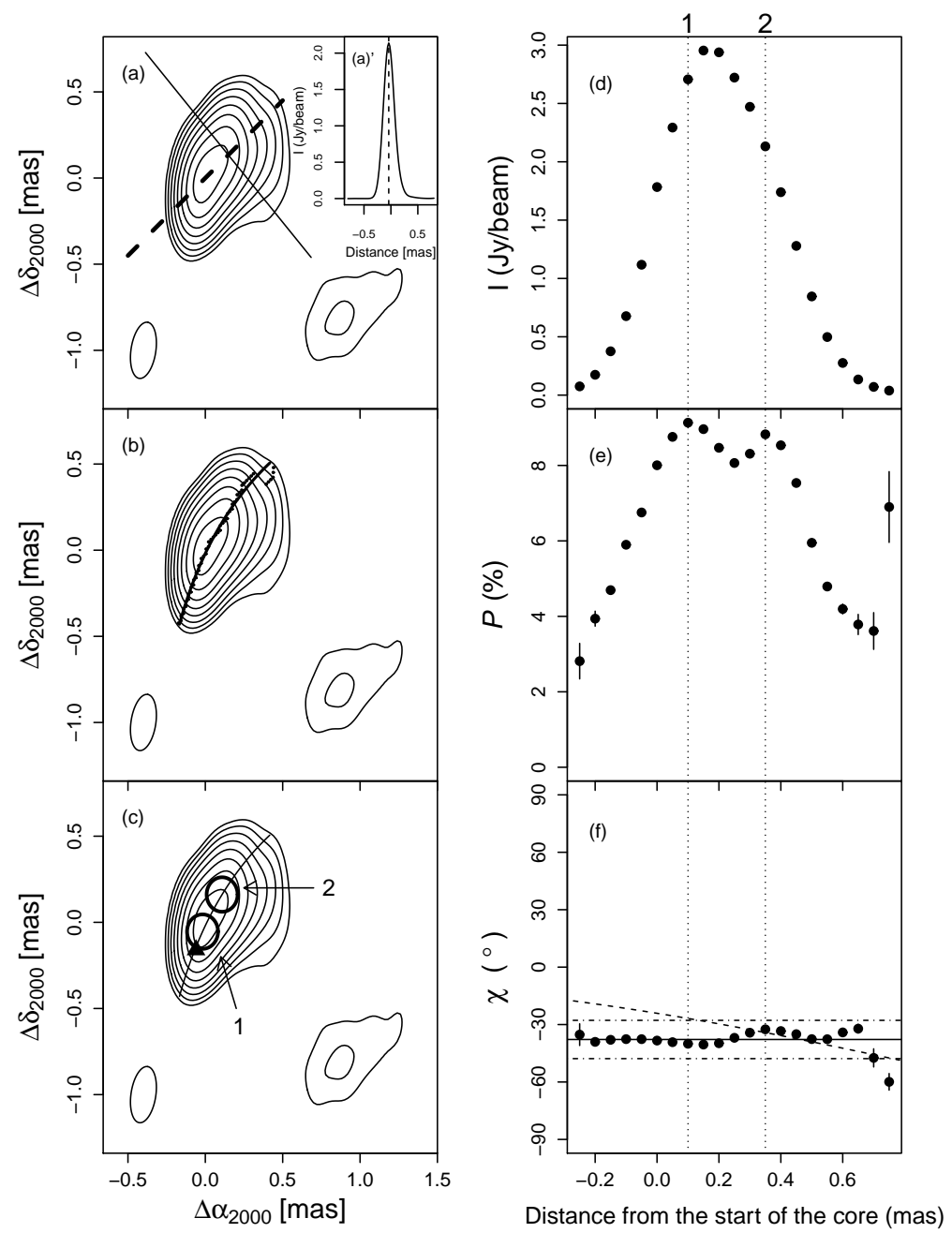

Figure 1. Example of VLBA images at $43 \mathrm{GHz}$ and profiles of intensity and polarization along the OJ 287 jet obtained on 2010 November 1. Restoring beam corresponds to ellipse in lower left corners of panels (a)-(c). Contour levels represent 0.5, 1, $2,4,8,16,32$, and $64 \%$ of the peak intensity of $3.13 \mathrm{Jy} /$ beam. Dashed line in panel (a) delineates rough jet direction. Intensity profile along the solid line is shown in panel (a) ${ }^{\prime}$ as an example of how trajectory points are determined (see text). Black circles in panel (b): maximum-intensity position at each radial distance; solid curve; best functional fit. Black triangle in panel (c): defined upstream boundary of the core. Panel (c) divides main emission structure into two circular Gaussian components with radii of 0.1 mas whose FWHM contours are indicated: (1) "core" and (2) stationary feature "S," with centers indicated by dotted lines in panels (d)-(f). Panels (d)-(f) show profiles of intensity, polarization, and EVPA along jet trajectory. Solid, dash dot, and dashed lines in panel (f) indicate, respectively, optical EVPA, $\pm 10^{\circ}$ areas of optical EVPA, and PA of jet trajectory.

as also found by Agudo et al. (2011) and Hodgson et al. (2017).

\subsection{Definition of Coincidence between the Optical and Radio EVPAs}

If the optical and radio emissions are co-spatial, then the EVPA of the radio emission should be similar to the optical EVPA. However, there is also the possibility of a chance coincidence between the varying radio EVPA in a more extended radio-emitting region and the observed optical EVPA. To assess the extent to which such spurious coincidences affect our results, we evaluate the systematic uncertainty of a chance coincidence between a random radio EVPA and the optical value. We generate 1000 uniform random numbers representing the radio EVPA ranging from $0^{\circ}$ to $180^{\circ}$. These numbers are subtracted from 1000 other random numbers selected from a normal distribution having the same mean as the observed optical EVPAs and a standard deviation equal to its uncertainty. The distribution of these differences should be related to the probability of chance coincidence between the optical and random radio EVPAs. We also estimate the distribution of differences between 
the random numbers selected from normal distributions having the same means as the observed optical and radio EVPAs. We define EVPA-coinciding positions as those where the differences between the radio and optical EVPAs are less than $10^{\circ}$, which is the standard deviation of random fluctuations in the optical EVPA over 100200 day intervals when there are no systematic EVPA variations. If the number of differences within $10^{\circ}$ between the observed optical and radio EVPAs is at least three times larger than those between simulated EVPAs selected from the normal (optical) and uniform (radio) distributions, corresponding to 3 sigma confidence, we classify the coincidence between the observed optical and radio EVPAs as significant. We evaluate the coincidence between the radio EVPAs at each position along the jet trajectory and optical EVPAs in this manner.

Positions of low $(<100)$ polarized intensity signal-tonoise ratios are excluded from the analysis. Applying this procedure to all 43 epochs, we find at least one EVPA-coinciding position along the jet trajectory in 36 of the epochs.

\section{RESULTS}

\subsection{Correlation between Radio and Optical Polarizations}

In the appendix we show all of the estimated profiles of the intensity, degree of polarization, and EVPA along the jet trajectory. We determine approximate values of these quantities for the core and stationary feature $S$ from the profiles. The profiles varied in time, as shown in Figures 6 - 9. In addition, the degree of polarization at most epochs varied with distance from the core, sometimes exceeding 10\%. At some epochs the EVPAs were constant, and at other epochs varied, with distance from the core.

Figure 2 shows a time series of the observed optical flux and polarization, as well as radio fluxes and polarization at both the core (at 0.05 mas) and $S$ (at $0.25 \mathrm{mas}$ ) estimated from individual profiles of the intensity, degree of polarization and EVPA.

Figure 3 plots the optical versus $7 \mathrm{~mm}$ degree of polarization at the position of peak total intensity in the VLBA image, measured at the same epoch. The degree of optical polarization is mildly correlated with that at $7 \mathrm{~mm}$, although the optical values tend to be higher. At only 18 epochs out of 43 is the $7 \mathrm{~mm}$ EVPA at the location of the total intensity peak within $\pm 10^{\circ}$ of the optical value. There are, however, 18 other epochs at which the optical and $7 \mathrm{~mm}$ EVPAs coincide within $10^{\circ}$ at positions other than the total intensity peak (see below); these are denoted by filled circles outside the dotted lines in the right panel of Figure 3. This implies that the primary site of optical emission can lie outside the brightest feature (usually the core) in the $7 \mathrm{~mm}$ image.

Most of the epochs have multiple positions along the jet at which the radio and optical EVPAs have similar orientations. One can consider two possibilities: (1) the optical emission originates from multiple locations along the jet, or (2) the magnetic field is systematically ordered along the jet. On the other hand, at seven epochs there are no coinciding positions. At some of these epochs the object was in an active state and at others it was in a quiescent phase. If the optically emitting region is optically thick at the radio frequency, the optical emission should not have a relationship with the (more extended) radio jet. The other possibility is that the radio polarization coming from the optically emitting region is diluted, with its mean changed by superposition of various polarization vectors associated with other regions.

The upper panel of Figure 4 shows the $7 \mathrm{~mm}$ intensity profile along the jet trajectory, averaged over all 43 epochs. We model the profile with two point components to estimate the separate intensity profiles of the core and $S$. The model with minimum $\chi^{2}$ value corresponds to average flux densities of the core and $S$ of 1.71 and $2.14 \mathrm{Jy}$, respectively, with a separation of 0.20 mas along PA $-28^{\circ}$. The solid curve in Figure 4 shows the fit of the model to the data (solid circles). The small deviations suggest that both the core and $S$ are slightly resolved rather than point-like sources.

As seen in Figure 4, the core dominates the intensity up to a distance of 0.13 mas, while $S$ dominates from 0.13 to 0.5 mas. The bottom panel of the figure demonstrates that the position along the jet where the optical and $7 \mathrm{~mm}$ EVPAs most closely correspond is sometimes associated with the core and other times with $S$. Some of the coinciding positions are in regions outside the core and $S$. If there is no relationship between the optical and radio EVPAs, the possibility of chance coincidence can be estimated from the fraction of EVPA differences that exceed the threshold of $10^{\circ}$. This criterion indicates that the optical and radio EVPAs at 6 out of 43 epochs can be expected to be similar by chance coincidence. Fewer than 5 coincidences are expected to occur in the regions outside the core and $S$, but this is too many to determine whether any optical emission comes from those regions. Therefore, the optical emission originates from either the core or stationary component $S$ (or both) at the majority of the epochs at which the optical and radio EVPAs are aligned.

\subsection{Differences between EVPAs and Jet Position Angle}




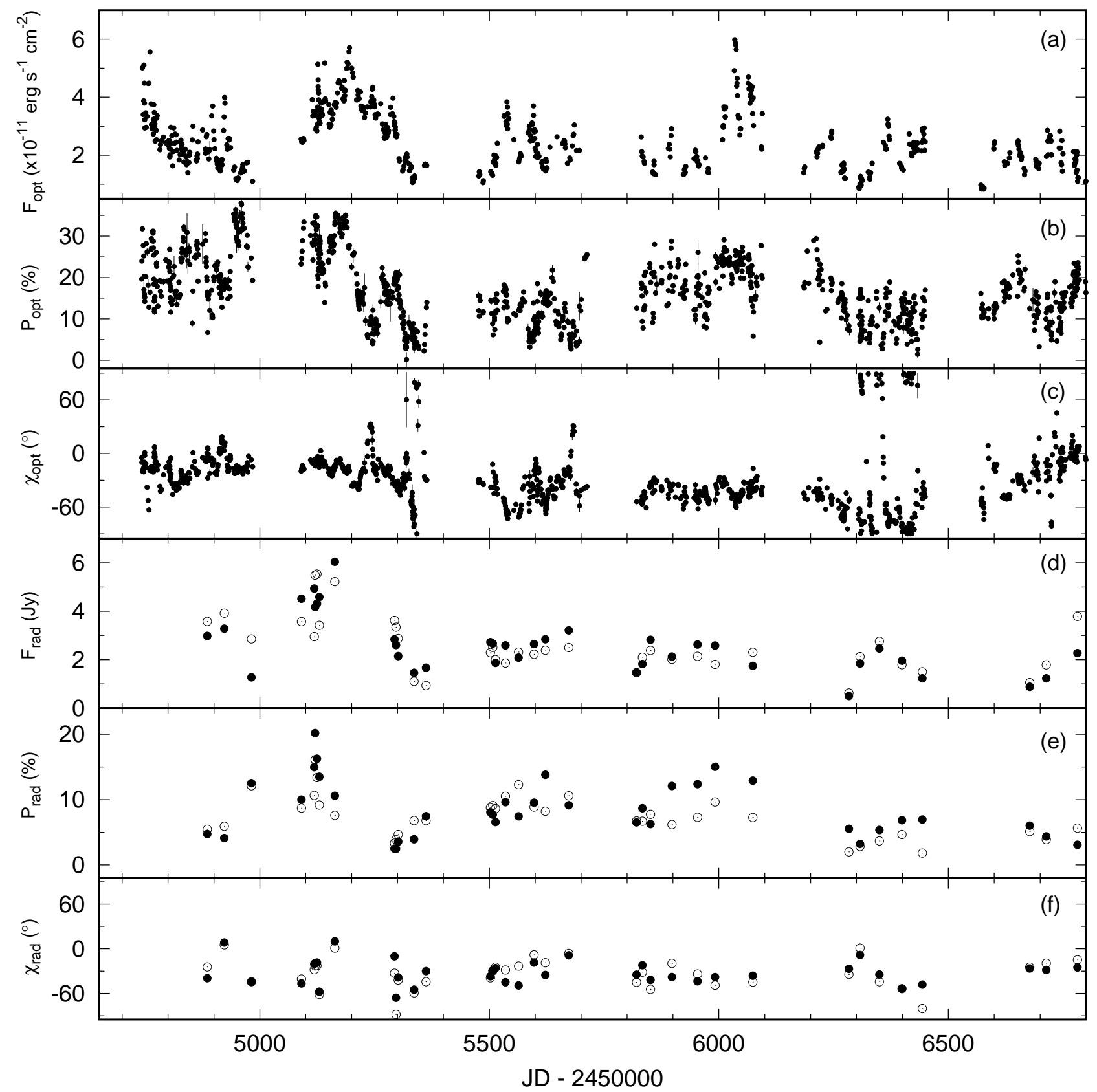

Figure 2. Time series of OJ 287; panel (a) - total intensity, panel (b) - degree of polarization, panel (c) - EVPA in the optical band. Panels (d), (e), and (f) show the corresponding time series of total intensity, degree of polarization and EVPA, for the measurements at 0.05 mas (open circles) and 0.25 mas (filled circles) where the "core" and the stationary feature $S$ are expected to be dominated. 

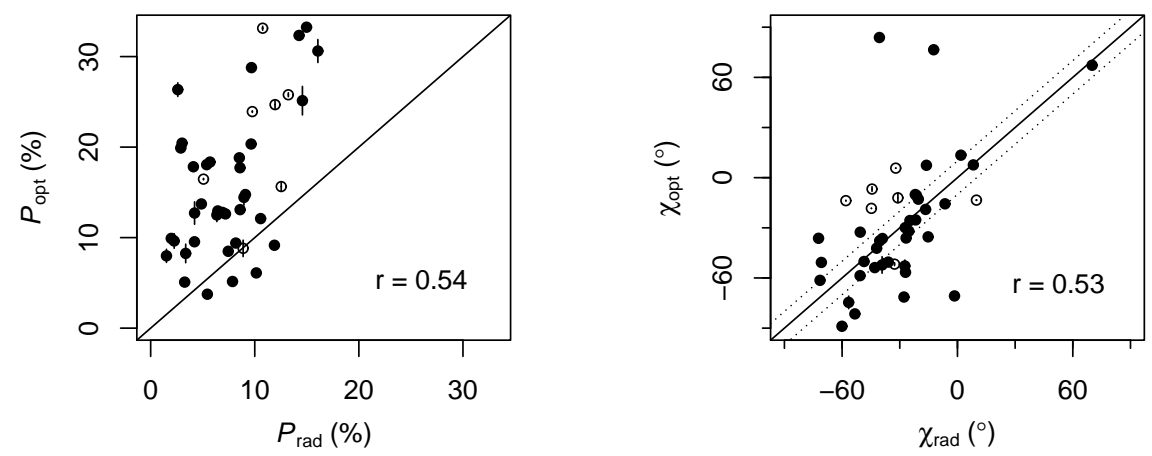

Figure 3. Optical vs. $7 \mathrm{~mm}$ degree of polarization (left) and EVPA (right). The $7 \mathrm{~mm}$ values are measured at location of peak total intensity. Solid line corresponds to equal optical and radio values; in right panel, dotted lines encompass EVPA values coinciding within $\pm 10^{\circ}$. Filled circles: cases when the $7 \mathrm{~mm}$ EVPA at one or more positions along the jet trajectory agrees with the optical EVPA within $\pm 10^{\circ}$; open circles: there are no such positions of similar EVPAs. Correlation coefficients are shown in bottom right of each panel. 


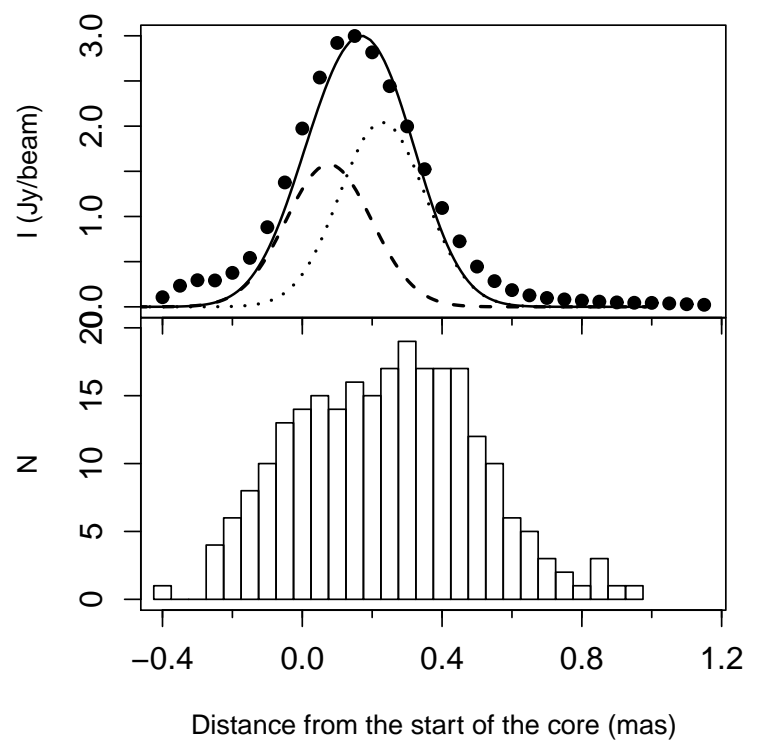

Figure 4. Top panel: Intensity profile along the jet trajectory averaged over all epochs considered here (filled circles). Curves represent the best-fit double point component model (dashed: core, dotted: stationary component $S$, solid: summed intensity). Bottom panel: Distribution of distances (measured from the upstream boundary of the core) of positions where the optical and $7 \mathrm{~mm}$ EVPAs are closest in value (only including those that coincide within $\pm 10^{\circ}$ ).

Figure 5 displays the distributions of differences between the PA of the jet trajectory and the EVPAs in cases where the optical and $7 \mathrm{~mm}$ EVPAs most closely correspond to each other (a) in feature $S$ and (b) in the core. Most of the EVPA-PA differences within $S$ are $<30^{\circ}$ (skewness score of 2.15), while those within the core region are more broadly distributed (skewness score of 1.34), although most are $<30^{\circ}$.

D'Arcangelo et al. (2009) measured EVPA values in the $7 \mathrm{~mm}$ core in 2006 that were perpendicular to the previously observed parsec-scale jet direction. This caused those authors to propose a spine-plus-sheath structure with different magnetic field geometries. However, as cited above, the jet changed direction abruptly by $\sim 90^{\circ}$ shortly before those observations. As a consequence, the core EVPA in 2006 was in fact nearly parallel to the new jet direction, a condition that continued with the observations reported here.

\section{DISCUSSION}

Our finding that optical synchrotron emission from OJ 287 arises in both the core and stationary feature $S$ agrees with the conclusions by Agudo et al. (2011) and Hodgson et al. (2017), based on timing of multi-

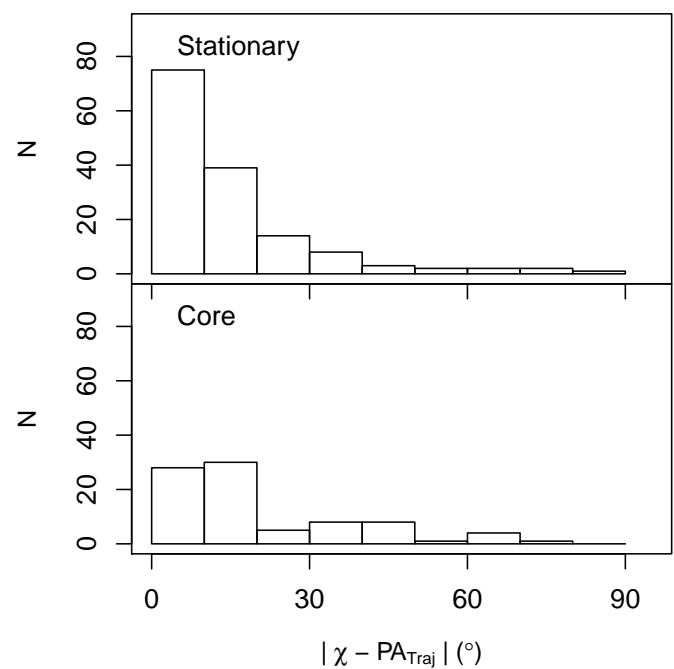

Figure 5. Distribution of differences between EVPA at $7 \mathrm{~mm}$ and local PA of jet trajectory at epochs when the optical and radio EVPAs match within $20^{\circ}$ inside either quasistationary feature $S$ (upper panel) or (b) the core region (lower panel). (Each 0.05 mas-radius region where the criterion is met is represented.)

waveband variations and motions of knot, that led to the conclusion that $\gamma$-ray and optical flares occur in both regions. This implies that both the core and $S$ accelerate electrons to $\mathrm{GeV}$ energies. The presence of optical emission downstream of the radio "core" has also been inferred by Kovalev et al. (2017) from displacements of the optical and radio centroids measured by Gaia and the VLBA.

The magnetic field geometry at the site of optical emission should relate to the main process(es) responsible for acceleration of GeV-energy electrons. Averaging of the polarization vector both across the line-of-sight and within the resolution beam can cause emission from turbulent regions - where second-order Fermi acceleration and magnetic reconnections can generate particle distributions extending up to $\mathrm{GeV}$ energies - to be weakly polarized, with random (in time) orientation of the EVPA. Instead, the polarization is quite strong in OJ 287 (Fig. 3) and avoids EVPAs $\gtrsim 50^{\circ}$ from the direction of the jet axis (Fig. 5). At some epochs, the optical and radio EVPAs are coincident at multiple locations, as in panel (f) of Figure 1. Since the radio polarization vectors are distributed along the jet, we cannot distinguish whether the optical emission comes from one narrow or multiple more diffuse regions.

The polarization produced by helical fields can be high or low, with EVPA that is usually parallel to the jet axis, 
although transverse EVPAs can arise for some viewing angles if the helix pitch angle is low (Lyutikov et al. 2005). Figure 5 shows that in OJ 287 the EVPA is nearly always within $30^{\circ}$ of the local jet direction. Reversals of magnetic field lines leading to reconnections can occur in sections of the jet where transverse velocity gradients shear magnetic loops into elongated patterns. In this case, the polarization would be high, with EVPAs perpendicular to the jet axis, contrary to the EVPAs in OJ 287.

A shock wave propagating down the jet, with the shock-normal oriented parallel to the jet axis, can cause strong polarization, with EVPA along the jet axis (Hughes et al. 1985). In OJ 287, however, we have identified the main emission as arising in two stationary features, the core and $S$. The beam-averaged polarization of turbulent plasma crossing a conical standing shock can be moderately high $(\sim 10-20 \%)$ with EVPA parallel to the axis (Cawthorne \& Cobb 1990). When resolved, the polarization vector has a more complex pattern, often roughly radial, centered near the position of peak intensity (Cawthorne 2006; Cawthorne et al. 2013). Agudo et al. (2011) have proposed that feature $S$ is such a standing conical shock.

In either the helical field or conical shock case, the EVPA of the net polarization can take on various values if there are asymmetries so that emission above or below the projected axis is higher than on the other side. This can occur, for example, because of turbulent fluctuations (Marscher 2014).

A less symmetric, nearly planar oblique shock should arise where the jet has impinged upon the external medium since it changed direction in 2005. However, such a shock would result in an EVPA that is very roughly transverse to the jet axis, contrary to the trend in OJ 287.

Particles can be accelerated by a relativistic shock wave in a collisionless plasma, with oblique (or conical) shocks favored over shocks oriented perpendicular to the jet axis (e.g., Summerlin \& Baring 2012; Baring et al. 2017). This results from the magnetic field lying closer to the shock-normal in the rest frame of the plasma (after taking into account relativistic aberration) and to the flow advecting away from the shock front more slowly than when the shock normal is parallel to the jet axis. These features allow the relativistic particles to cross the shock front multiple times. The accelerated $\mathrm{GeV}$-energy electrons lose energy via radiative cooling as they emit optical and (via inverse Compton scattering) $\gamma$-ray photons. The synchrotron cooling timescale is $\sim 2 \mathrm{~d}$ in the observer's frame for a magnetic field strength $\sim 0.1 \mathrm{G}$ and Doppler factor $D \sim 9$
(Tashiro et al. 1995; Sasada et al. 2010; Jorstad et al. 2017), hence rapid variability is possible. In this scenario, the optically emitting region lies in a thin layer close to the shock front where the magnetic field remains compressed (Marscher \& Gear 1985).

The jet of M87 is among the most well-studied jets among active galaxies and has a stationary feature known as HST-1. Avachat et al. (2016) find that the EVPAs in HST-1 are roughly along the jet direction at optical wavelengths, but nearly perpendicular at $22 \mathrm{GHz}$. In OJ 287, the similar behavior of the difference between the jet PA and the EVPAs at both wavebands suggests that the optical and radio polarization patterns are more similar.

\section{CONCLUSIONS}

Our comparison of the total optical polarization and the polarization along the jet axis in $7 \mathrm{~mm}$ VLBA images confirms the conclusion of Agudo et al. (2011) and Hodgson et al. (2017) that the optical emission in OJ 287 arises both in the "core" and the bright stationary feature $S$ located 0.2 mas downstream of the core. When the optical EVPA coincides with that at $7 \mathrm{~mm}$ within $S$, the polarization vector nearly always lies $<30^{\circ}$ from the direction of the jet axis. This is consistent with $S$ being a standing shock of the type that can accelerate electrons to energies high enough to radiate at optical and $\gamma$-ray frequencies.

Since feature $S$ is situated near a bend in the jet trajectory, its high intensity - comparable to and sometimes exceeding that of the core (Agudo et al. 2011) - implies that strong optical and $\gamma$-ray emission can result from such changes in jet direction. The ongoing VLBA-BU-BLAZAR monitoring program is well-suited to determine whether this possible connection between high-frequency emission and jet bending is common in blazars.

The authors thank Dr. Ioannis Myserlis for a critical review of a draft of this manuscript. MS was supported during this study by a JSPS Postdoctoral Fellowship for Research Abroad. The research at Boston University was supported in part by National Science Foundation grant AST-1615796 and NASA Fermi Guest Investigator Program grants NNX14AQ58G and 80NSSC17K0649. The St.Petersburg University team acknowledges support from Russian Science Foundation grant 17-1201029. IA acknowledges support by a Ramón y Cajal grant of the Ministerio de Economía y Competitividad (MINECO) of Spain. The research at the IAA-CSIC was supported in part by the MINECO through grants AYA2016-80889-P, AYA2013-40825-P, and AYA2010- 
14844, and by the regional government of Andalucía through grant P09-FQM-4784. Calar Alto Observatory is jointly operated by the Max-Planck-Institut für Astronomie and the IAA-CSIC. The VLBA is an instrument of the Long Baseline Observatory (LBO). The LBO is a facility of the National Science Foundation operated under cooperative agreement by Associated Universities, Inc. Data from the Steward Observatory spectropolarimetric monitoring project were used. This program was supported by Fermi Guest Investigator grants NNX08AW56G, NNX09AU10G, NNX12AO93G, and NNX15AU81G.

\section{APPENDIX}

Figures 6-9 present profiles of intensities, degrees of polarization and EVPAs along the jet trajectory at 43 epochs. Displayed from top to bottom at each epoch are the intensity, degree of polarization, and EVPA. In the bottom panel, the dashed line indicates the PA of the jet trajectory, while the solid and dotted lines mark the optical EVPA and the thresholds of $\pm 10^{\circ}$, respectively.

\section{REFERENCES}

Agudo, I., Marscher, A. P., Jorstad, S. G., et al. 2011, ApJL, 735, L10

Agudo, I., Marscher, A. P., Jorstad, S. G., et al. 2012, ApJ, 747,63

Agudo, I., Thum, C., Casadio, C., et al. 2018a, MNRAS, 474, 1427

Agudo, I., Thum, C., Ramakrishnan, V., et al. 2018b, MNRAS, 473, 1850

Avachat, S. S., Perlman, E. S., Adams, S. C., et al. 2016, ApJ, 832, 3

Baring, M. G., Böttcher, M., \& Summerlin, E. J. 2017, MNRAS, 464, 4875

Cawthorne, T. V., \& Cobb, W. K. 1990, ApJ, 350, 536

Cawthorne, T. V. 2006, MNRAS, 367, 851

Cawthorne, T. V., Jorstad, S. G., \& Marscher, A. P. 2013, ApJ, 772, 14

D'Arcangelo, F. D., Marscher, A. P., Jorstad, S. G., et al. 2009, ApJ, 697, 985

Gómez, J.-L., Marscher, A. P., Alberdi, A., Jorstad, S. G., \& Agudo, I. 2001, ApJL, 561, L161

Goyal, A. and Stawarz, L. and Zola, S. and Marchenko, V. and Soida, M. et al. 2017, arXiv170904457

Hada, K., Doi, A., Kino, M., et al. 2011, Nature, 477, 185

Hodgson, J. A., Krichbaum, T. P., Marscher, A. P., et al. 2017, A\&A, 597, A80

Hughes, P. A., Aller, H. D., \& Aller, M. F. 1985, ApJ, 298, 301

Ikejiri, Y., Uemura, M., Sasada, M., et al. 2011, PASJ, 63, 639

Jorstad, S. G., Marscher, A. P., Lister, M. L., et al. 2005, AJ, 130, 1418

Jorstad, S. G., Marscher, A. P., Stevens, J. A., et al. 2007, AJ, 134, 799

Jorstad, S. G., Marscher, A. P., Larionov, V. M., et al. 2010, ApJ, 715, 362
Jorstad, S. G., \& Marscher, A. P. 2016, Galaxies, 4, 47

Jorstad, S. G., Marscher, A. P., \& Morozova, D. A. 2017, ApJ, 846, 98

Joshi, M., \& Böttcher, M. 2011, ApJ, 727, 21

Kawabata, K. S., Okazaki, A., Akitaya, H., et al. 1999, PASP, 111, 898

Kovalev, Y. Y., Petrov, L., \& Plavin, A. V. 2017, A\&A, 598, L1

Larionov, V. M., Jorstad, S. G., Marscher, A. P., et al. 2008, A\&A, 492, 389

León-Tavares, J., Lobanov, A. P., Chavushyan, V. H., et al. 2010, ApJ, 715, 355

Lyutikov, M., Pariev, V. I., \& Gabuzda, D. C. 2005, MNRAS, 360, 869

Marscher, A. P., \& Gear, W. K. 1985, ApJ, 298, 114

Marscher, A. P. 2008, in Astronomical Society of the Pacific Conference Series, Vol. 386, Extragalactic Jets: Theory and Observation from Radio to Gamma Ray, ed. T. A. Rector \& D. S. De Young, 437

Marscher, A. P., Jorstad, S. G., D'Arcangelo, F. D., et al. 2008, Nature, 452, 966

Marscher, A. P., Jorstad, S. G., Larionov, V. M., et al. 2010, ApJL, 710, L126

Marscher, A. P., \& Jorstad, S. G. 2011, ApJ, 729, 26

Marscher, A. P. 2014, ApJ, 780, 87

Marscher, A. P. 2016, Galaxies, 4, 37

Nilsson, K., Takalo, L. O., Lehto, H. J., \& Sillanpää, A. 2010, A\&A, 516, A60

R Core Team. 2015, R: A Language and Environment for Statistical Computing, R Foundation for Statistical Computing, Vienna, Austria

Sasada, M., Uemura, M., Arai, A., et al. 2010, PASJ, 62, 645

Sironi, L., Petropoulou, M., \& Giannios, D. 2015, MNRAS, 450,183 

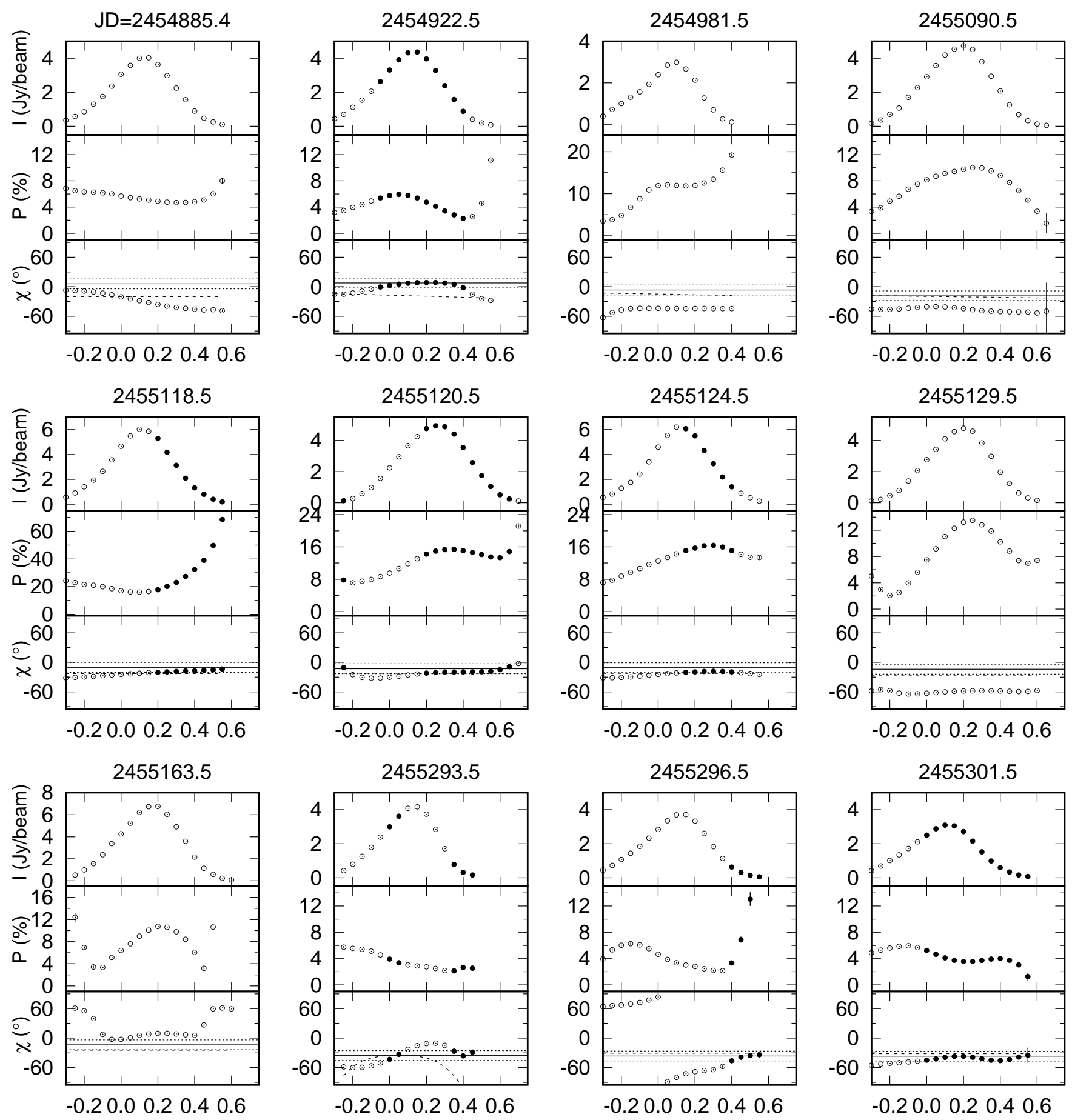

Figure 6. Profiles of intensites, degrees of polarization and EVPAs along the jet trajectory from the epoch of February 2009 to April 2010. 


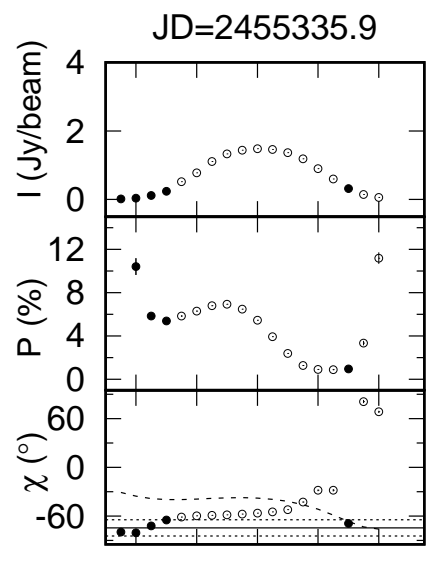

$\begin{array}{llll}-0.20 .0 & 0.20 .40 .6\end{array}$
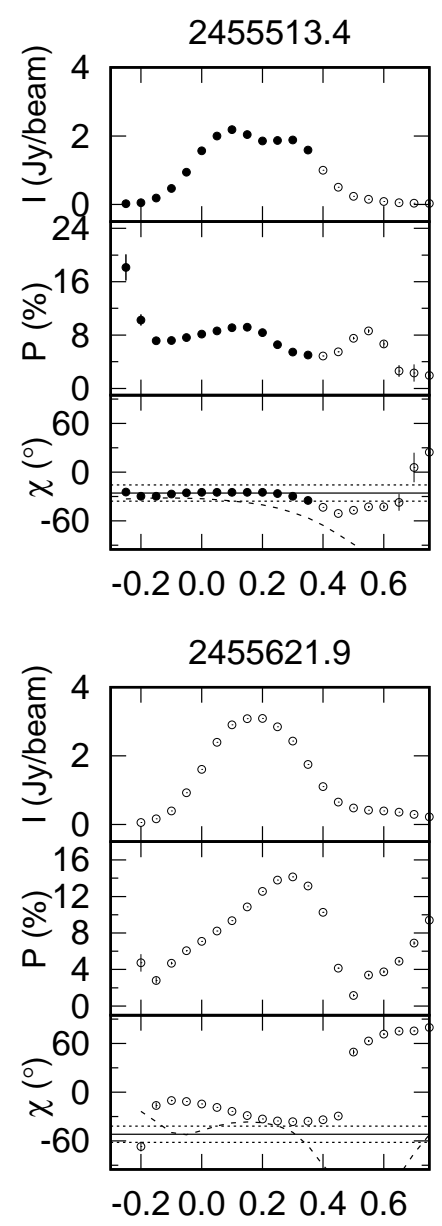
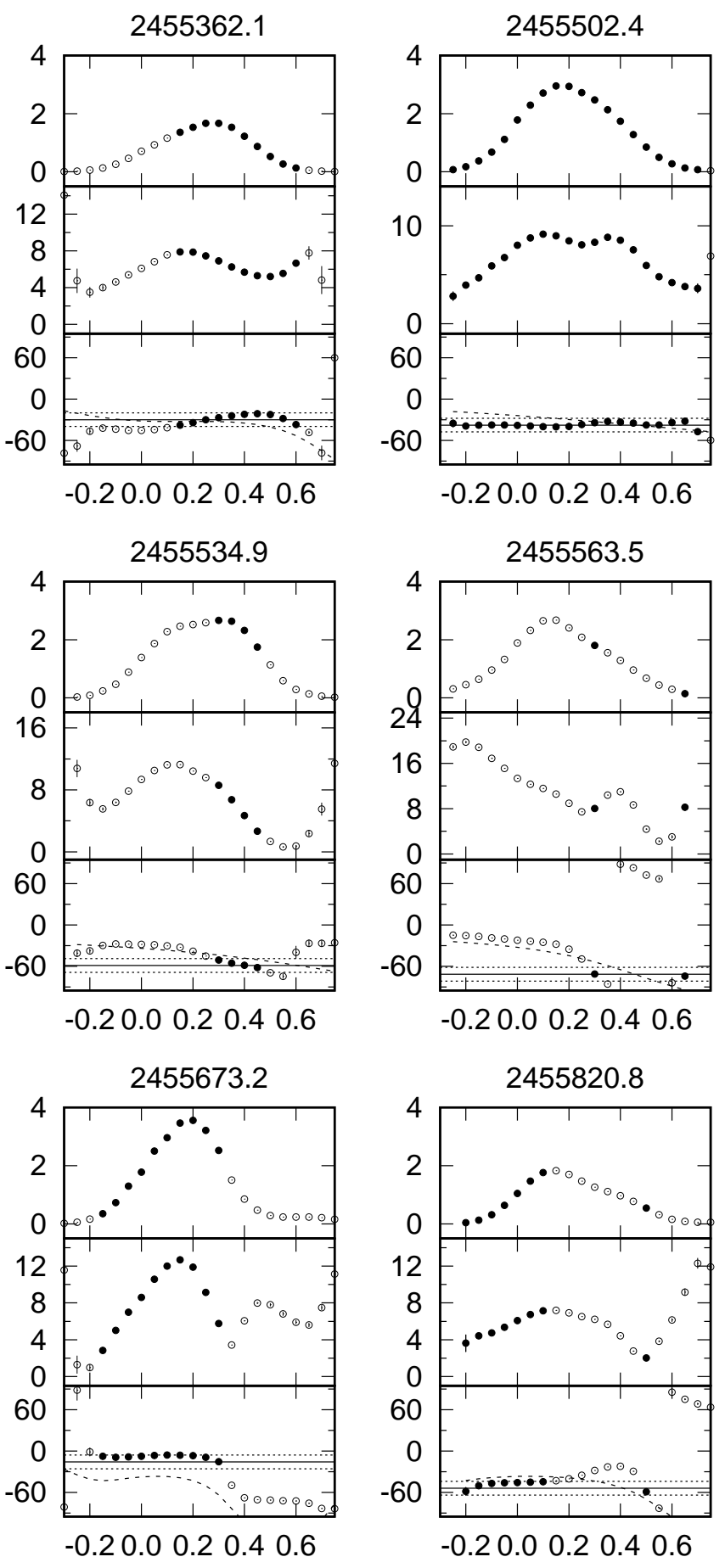
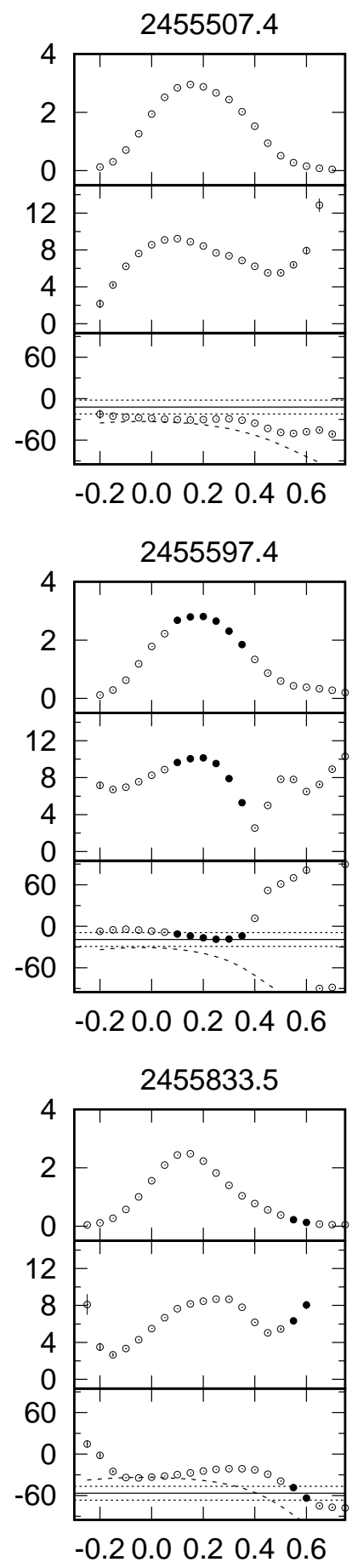

Figure 7. Epochs of May 2010 to September 2011. 

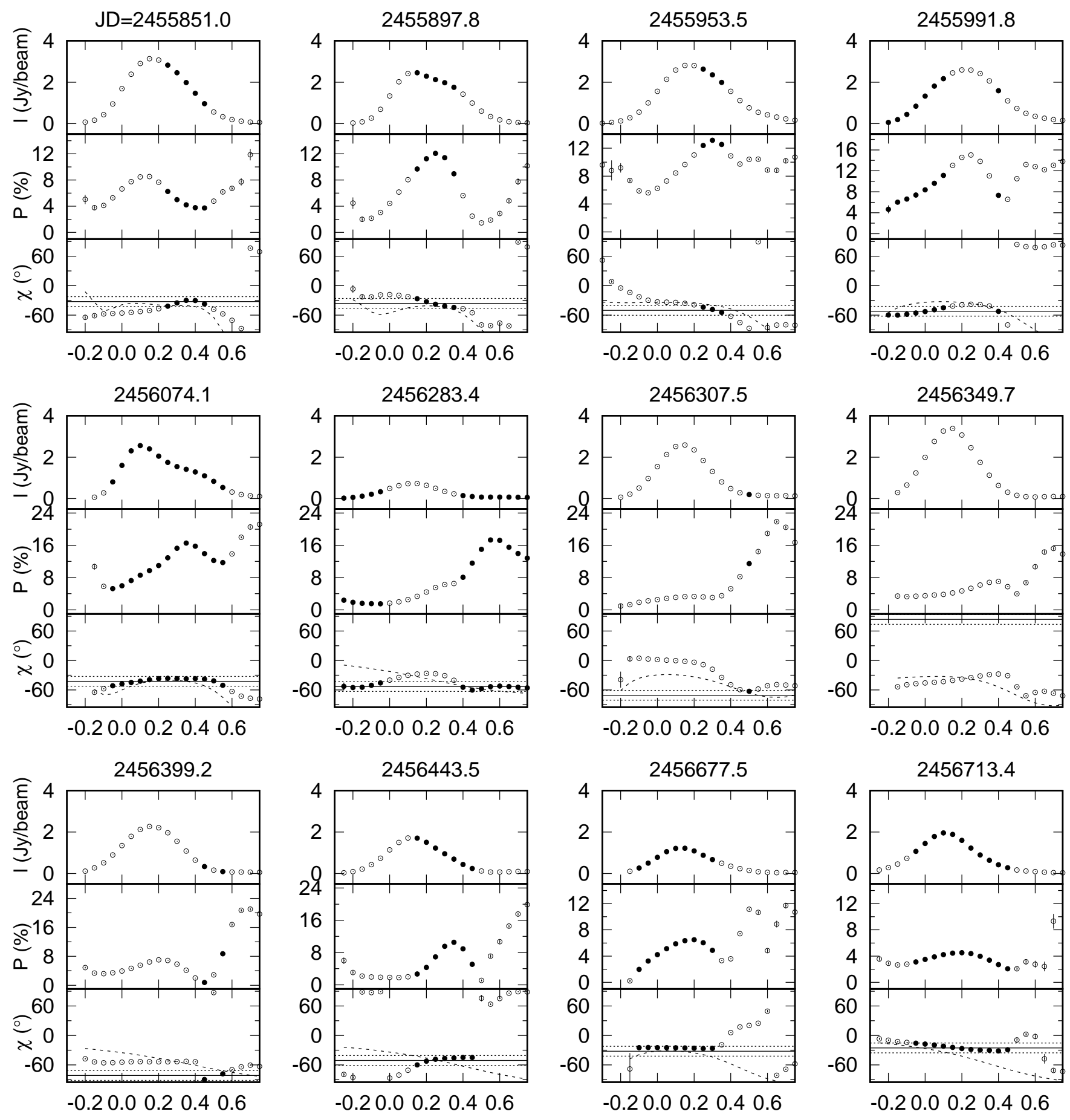

Figure 8. Epochs of October 2011 to February 2014. 

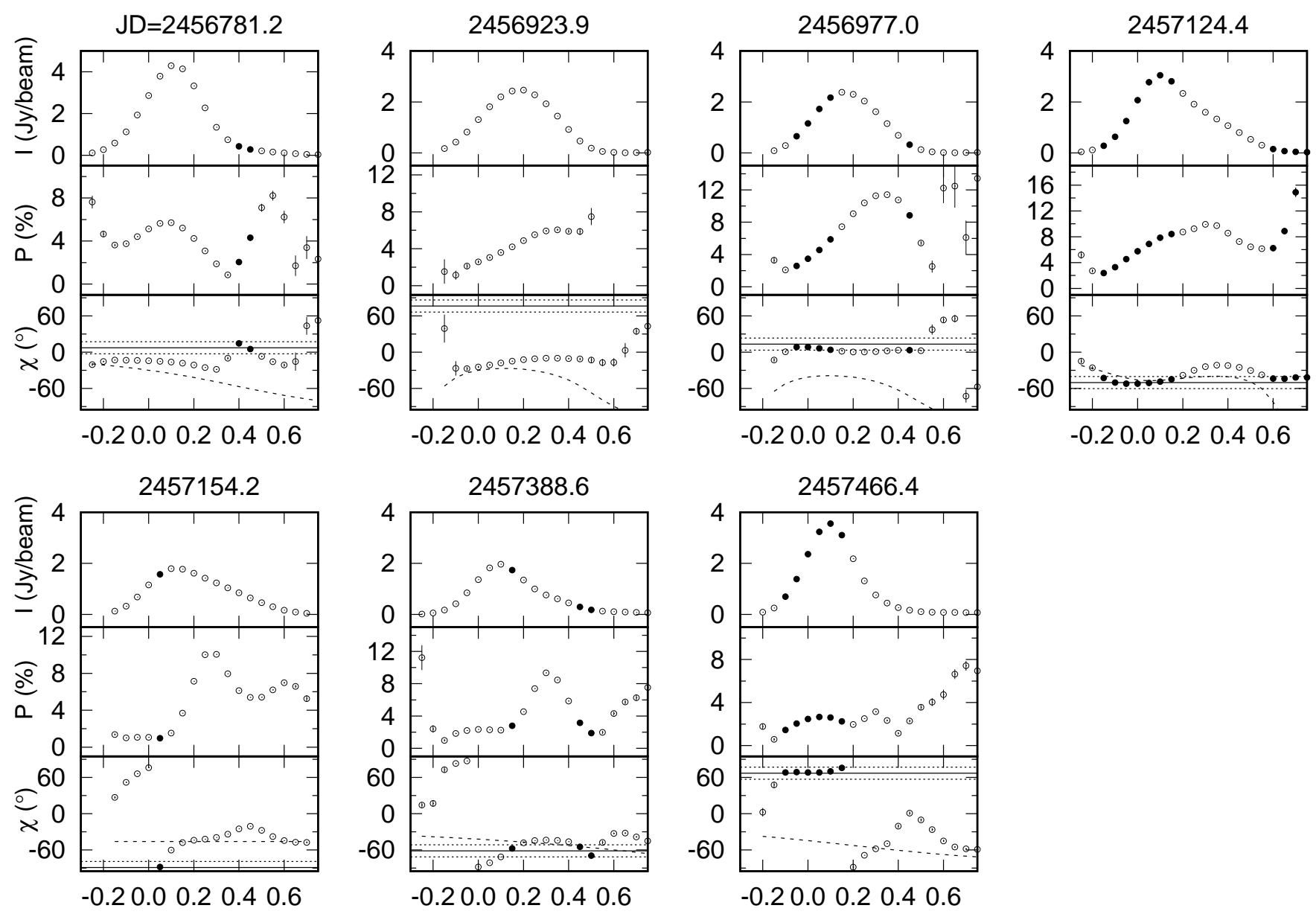

Figure 9. Epochs of May 2014 to March 2016. 
Smith, P. S., Montiel, E., Rightley, S., et al. 2009, ArXiv e-prints, arXiv:0912.3621

Summerlin, E. J., \& Baring, M. G. 2012, ApJ, 745, 63

Tashiro, M., Makishima, K., Ohashi, T., Inda-Koide, M., Yamashita, A., Mihara, T. \& Kohmura, Y. PASJ, 47, 131
Villforth, C., Nilsson, K., Heidt, J., et al. 2010, MNRAS, 402, 2087

Wardle, J. F. C., \& Kronberg, P. P. 1974, ApJ, 194, 249 